\title{
Experimental Investigation on Multiscale Fracturing in Thermally Treated Sandstone under SHPB Impact Loading
}

\author{
Shang Yang, ${ }^{1}$ Jun Wang $\mathbb{D}^{1,2}$ Zhi Zhang, ${ }^{1}$ Jianguo Ning, ${ }^{1}$ and Guoqing Sun ${ }^{1}$ \\ ${ }^{1}$ College of Energy and Mining Engineering, Shandong University of Science and Technology, Qingdao 266590, China \\ ${ }^{2}$ State Key Laboratory of Mining Disaster Prevention and Control Co-founded By Shandong Province and the Ministry of Science \\ and Technology, Shandong University of Science and Technology, Qingdao 266590, China
}

Correspondence should be addressed to Jun Wang; wangjunsdkjd@126.com

Received 11 September 2021; Accepted 27 October 2021; Published 24 November 2021

Academic Editor: Zhanbo Cheng

Copyright (C) 2021 Shang Yang et al. This is an open access article distributed under the Creative Commons Attribution License, which permits unrestricted use, distribution, and reproduction in any medium, provided the original work is properly cited.

To study the effect of temperature on the dynamic mechanical behaviors and fracture characteristics of thermally treated sandstone, compressive dynamic loading experiments were performed on a series of samples using a split Hopkinson pressure bar (SHPB). In the tests, the dynamic uniaxial compressive strength of the thermally treated sandstone was inversely proportional to the temperature treatment in the range of $200^{\circ} \mathrm{C}-1000^{\circ} \mathrm{C}$, while the dynamic elastic modulus first increased and then gradually decreased after different temperature treatments. The results show that two classical mechanical types (i.e., Class I and Class II) are observed from the dynamic stress-strain responses of SHPB tests for thermally treated sandstone. By means of scanning electron microscopy (SEM), the microdifference of postloading microfracture characteristics in Class I and Class II behavior was identified. In Class I behavior, intercrystalline cracks (IE) are the chief form of cracks on the fracture surface of a specimen fractured by SHPB loading even though there some intracrystalline cracks may also be present. In contrast, Class II behavior results from the chief cracking type being intracrystalline cracks.

\section{Introduction}

In recent decades, an increasing number of rock engineering projects, such as deep mining of mineral resources $[1,2]$, borehole drilling in tunnel excavation [3], and geothermal resource exploitation [4], have been encountered in hightemperature environments. As the temperature increases, the physical-mechanical properties of rocks change [5-10], and a series of difficult problems arise that jeopardize the stability and safety of rock engineering projects.

To investigate the failure process of thermally treated rock, many experiments have been carried out, and a great deal of valuable findings was published. Wong and Brace [11] found that the thermal expansion of rock was irreversible and proposed theoretical models to explain the effects of temperature on thermal expansion. In addition, the thermally treated rock properties at different scales at the rock statics domain level were discussed, such as rock permeability, rock burst tendency, acoustic emission, $\mathrm{P}$-wave velocity, bearing capacity, and deformation modulus [12-15]. At a microlevel, Ding et al. [16] found that the microcracks inside thermally treated sandstone increase when the temperature exceeds $400^{\circ} \mathrm{C}$ using scanning electron microscopy (SEM). Yang et al. [17] pointed out that the damage of granite samples increased with increasing thermally treated temperature. Ferrero and Marini [18] studied the behavior of two types of marble after high-temperature treatments of up to $600^{\circ} \mathrm{C}$ using microscopic analyses and open porosity tests; they found that new cracks formed and that open porosity increased with increasing temperature. Xiong et al. [19] studied the mechanical properties of artificial jointed rock mass specimens with a single joint plane after high temperatures; the results show that the uniaxial compressive strength of the specimen is lowest when the dip angle of the artificial joint plane is $60^{\circ}$. However, considering previous work at the rock statics domain level, 
understanding the deformation and failure characteristics of thermally treated rocks that have been subjected to dynamic loading is sometimes challenging.

Various experiments have been conducted on the mechanical properties and failure behavior of nonthermally treated rocks that were subjected to dynamic loading. Previous studies [20-23] show that under dynamic loading, the strain rate, confining pressure, and temperature influence rock mechanical properties. For example, Li et al. [24] noted that failure mechanisms can be categorized into two classical mechanical types: Class I and Class II. At a lower strain rate, mechanical Class I samples are primarily subject to elastic deformation, and the sample generally does not fail. At higher strain rates, Class II samples are qualitatively described as pulverized. The dynamic strength has a nonlinear increase in the strain rate at the full scope. Wong et al. [25] carried out dynamic uniaxial compression tests of Carrara marble held at various temperatures using an SHPB and noted that the typical stress-strain curve is a mixture of Class I and Class II fracturing. They also found that the dynamic strength of the marble specimen decreased with heating temperature. Liu and Xu [26] concluded that the dynamic mechanical properties of marble after heating changes due to the transformation of mineral components and the change in mineral particles. In addition, Chen et al. [27] conducted a study on failure modes of post-high-temperature sandstone by an SHPB system and found that the break degree increased with increasing temperature. However, the performance of adequate studies requires that the investigation of the temperature effects on rock dynamic mechanical behavior considers the microcharacteristics of crack fracturing in detail. Therefore, the microdifference of rock microcracks must be studied under SHPB impact loading.

In this study, typical thermally treated sandstone was selected for tests with dynamic loading conditions using an SHPB apparatus. The differences in rock failure modes and peak stress were analyzed and compared systematically. In addition, the postloading microfracture characteristics of the specimens were studied by scanning electron microscopy. The purpose was to identify the rules of rock dynamic mechanical behavior and examine the microfracture characteristics of a sample section.

\section{Description of Medium Sandstone}

Medium sandstone was obtained from the Jinhuagong coal mine in Datong, Shanxi Province, China. A mineral component analysis of the medium sandstone indicated (Figure 1) that the modal composition is $33 \%$ quartz (with a grain radius of $0.2-0.45 \mathrm{~mm}$ ), $21 \%$ feldspar (with a grain radius of $0.25-0.35 \mathrm{~mm}$ ), $34 \%$ debris (which primarily consisted of igneous debris), and 12\% agglutinate (which consists of kaolinite, microcrystalline carbonate, and reticulated clay). These specimens were used for various laboratory tests, as explained in the following sections.

\section{Experimental Methodology}

3.1. Specimen Preparation. To weaken the structural heterogeneity influence on the discreteness of the experimental results, all the tested samples were taken from the same block

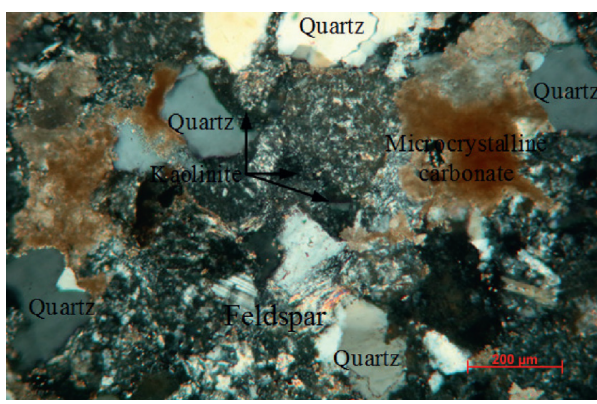

Figure 1: Optical microscopy of medium sandstone.

with no visible geological weakness. The length to diameter ratios were set as $1: 1$ to reduce the inertia effect, as well as the friction constraint. Following the ISRM suggestion [28], all test specimens were polished carefully at both ends to ensure that no perpendicularity was less than $0.02 \mathrm{~mm}$. Moreover, to best satisfy the assumption of uniformity, all test specimens were polished carefully through the machine to ensure that no perpendicularity was less than $0.02 \mathrm{~mm}$ and that the perpendicularity to the axis of the specimen was within $0.25^{\circ}$. The mechanical parameters, such as uniaxial compressive strength (with a mean of $76.6 \mathrm{MPa}$ ), Young's modulus (with a mean of $6.73 \mathrm{GPa}$ ), and Poisson's ratio (with a mean of 0.28 ), were obtained by the static uniaxial compression test.

\subsection{Experimental Apparatus Testing Program. All laboratory} tests were carried out in a modified SHPB loading system, as shown in Figure 2. It consists of a striker launcher, an incident bar, a transmitted bar, an absorbing bar, a damper, an axial prestress component, and a data collection and recording device. The striker launcher (Figure 2(c)) is used to generate a stress pulse, which consists of a cone-shaped striker, a gas gun, a high-pressure nitrogen tank, and a gas pressure control device. The cone-shaped striker was made of $40 \mathrm{Cr}$ alloy of $50 \mathrm{~mm}$ in diameter and $360.1 \mathrm{~mm}$ in length, and its geometry is shown in Figure 2(a). Incident, transmitted, and absorbing bars were also made of high-strength $40 \mathrm{Cr}$ alloy. Measurements showed that the P-wave velocity, elastic modulus, and density of the three bars were $5400 \mathrm{~m} / \mathrm{s}$, $240 \mathrm{GPa}$, and $7810 \mathrm{~kg} / \mathrm{m}^{3}$, respectively. According to international standards, the incident bar and transmitted bar were $50 \mathrm{~mm}$ in diameter and aligned with the center of the absorbing bar. The incident and transmitted bars were $3.0 \mathrm{~m}$ in length, but the absorbing bar was $1.5 \mathrm{~m}$ in length.

The data collection and recording device consists of pairs of resistance strain gauges, an ultrahigh dynamic strain meter (SDY2107 A), and a central console. It can not only receive, store, and display the stress pulse of the incident and transmitted bars but also measure the velocity of the cone-shaped striker. The resistance, length, and gauge factor of the resistance were $120 \Omega, 10 \mathrm{~mm}$, and 2 , respectively. It was stick to incident and transmitted bars. SDY2107 A covered 10 channels with a frequency response of $2.5 \mathrm{kHz}$ and was used for dynamic strain measurement. In addition, measurements were made using the standard software DS-750 supplied to the DS-50A. 


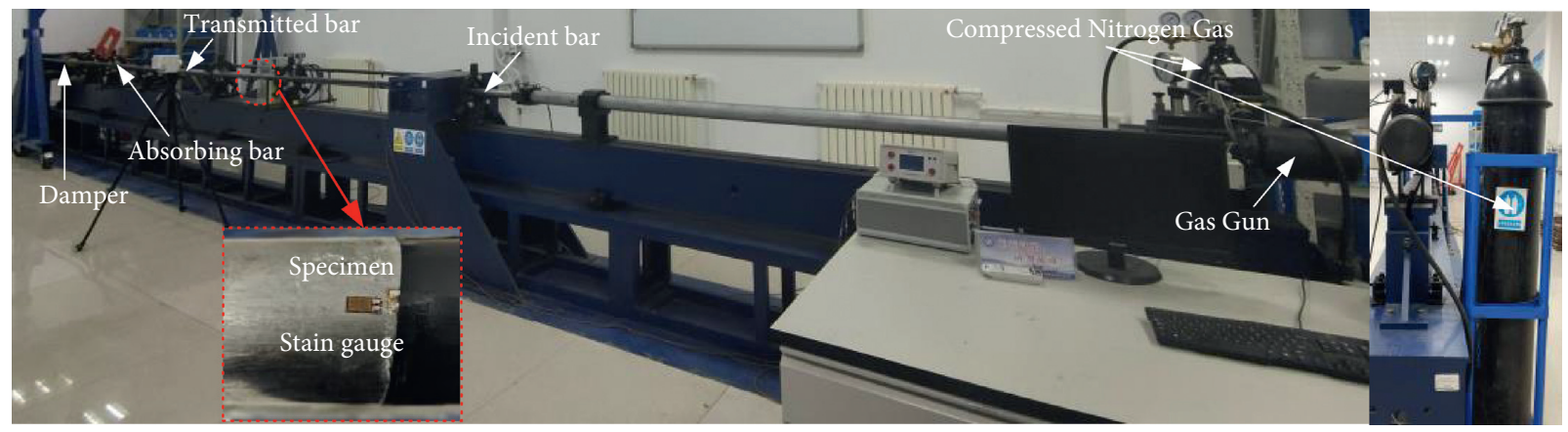

(a)

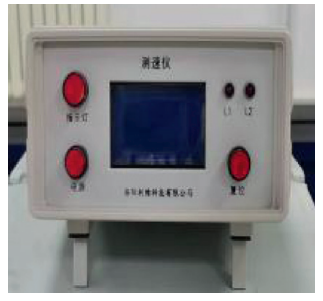

Velocimeter

(b)

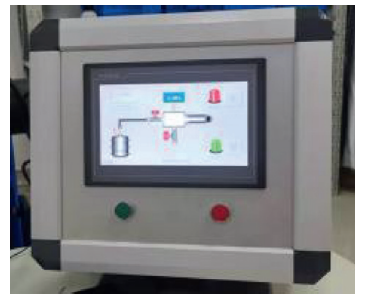

Gas pressure control device

(c)

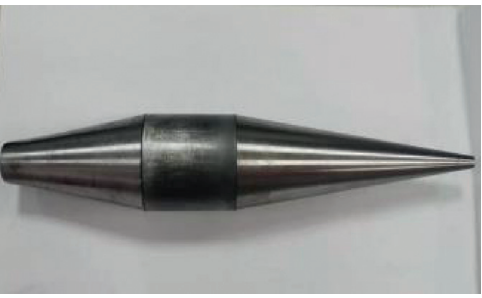

Cone-shaped striker

(c)

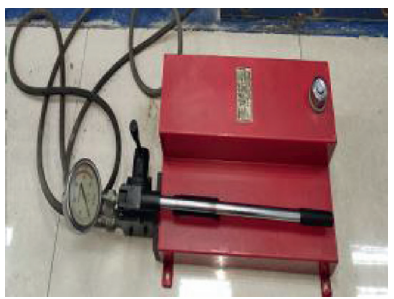

Axial prestress component

(d)

Figure 2: Diagram of the SHPB experiment system.

3.3. Testing Procedure. In this study, the wave velocity of the samples was measured using a ZBL-U520 nonmetal detector. Then, 30 samples with a velocity of $3.3 \mathrm{~km} / \mathrm{s}$ were selected. The selected samples were divided into six groups, with three samples in each group. Each group was exposed to room temperature or heated to high temperature at $200^{\circ} \mathrm{C}$, $400^{\circ} \mathrm{C}, 600^{\circ} \mathrm{C}, 800^{\circ} \mathrm{C}$, and $1000^{\circ} \mathrm{C}$ using a QSH-1200 T boxtype high-temperature furnace. Heating the samples to the predetermined temperature, three stages were as follows: first, the samples were heated in a furnace at a heating rate of $15{ }^{\circ} \mathrm{C} / \mathrm{min}$; second, to ensure that the samples were sufficiently heated, each designated temperature was kept constant for $24 \mathrm{~h}$; and finally, to avoid thermal shocks, the specimens were cooled naturally to room temperature $\left(25^{\circ} \mathrm{C}\right)$ in the furnace.

For the purpose of this study, we performed conventional dynamic uniaxial compression experiments at a modified SHPB loading system, and the petroleum jelly was applied on the ends of the specimen to weaken the end friction effects in this experiment. The striker was launched from the gas gun with the same velocity and impacted the front end of the incident bar. Then, the strain rate $\left(56.7 \mathrm{~s}^{-1}\right)$ was relatively stable in this study.

\subsection{Data Processing}

3.4.1. Calculation of the Strain and Stress. Conventional dynamic uniaxial compression tests assumed one-dimensional elastic wave propagation in the incident and transmitted bars and homogeneous deformation of the sample [28]. During the tests, three elastic stress pulses captured by pulses from the strain gauge, incident strain, reflected strain, and transmitted strain were extracted. On the basis of the one-dimensional stress wave propagation theory, the three-wave analysis method was used to determine the stress-strain curve of the specimen. Using incident, reflected, and transmitted strain pulses, the axial stress $\sigma(t)$, strain $\varepsilon(t)$, and strain rate $\dot{\varepsilon}(t)$ of the specimen were derived as follows:

$$
\begin{aligned}
& \sigma(t)=\frac{A_{0} E_{0}}{2 A_{s}}\left[\varepsilon_{i}(t)+\varepsilon_{r}(t)+\varepsilon_{t}(t)\right], \\
& \varepsilon(t)=\frac{c_{0}}{l_{0}} \int_{0}^{t}\left[\varepsilon_{i}(t)+\varepsilon_{r}(t)+\varepsilon_{t}(t)\right] d \tau, \\
& \dot{\varepsilon}(t)=\frac{c_{0}}{l_{0}}\left[\varepsilon_{i}(t)-\varepsilon_{r}(t)-\varepsilon_{t}(t)\right],
\end{aligned}
$$

where $A_{0}, C_{0}$, and $E_{0}$ are the cross-sectional area, P-wave velocity, and Young's modulus of the elastic bar, respectively, and $A_{s}$ and $L_{s}$ are the cross-sectional area and length of the specimen, respectively.

Thus, the stress, strain, and strain rate histories of specimens subjected to coupled static-dynamic compression can be obtained using the three-wave analysis method.

3.4.2. Dynamic Stress Equilibrium. For SHPB tests, a dynamic force balance between the ends of the specimen should be approximately achieved prior to the peak stress in the specimen, which can minimize the axial inertial effect. According to ISUM's suggestion [20], the pulse-shaping technique was adopted to achieve a dynamic force balance. In this study, a cone-shaped striker was also used to generate a ramped (half sine) incident wave to achieve a dynamic force balance at the ends of the specimen. The dynamic stress balance can be checked by comparing the incident, reflected, and transmitted stress histories during the test. 


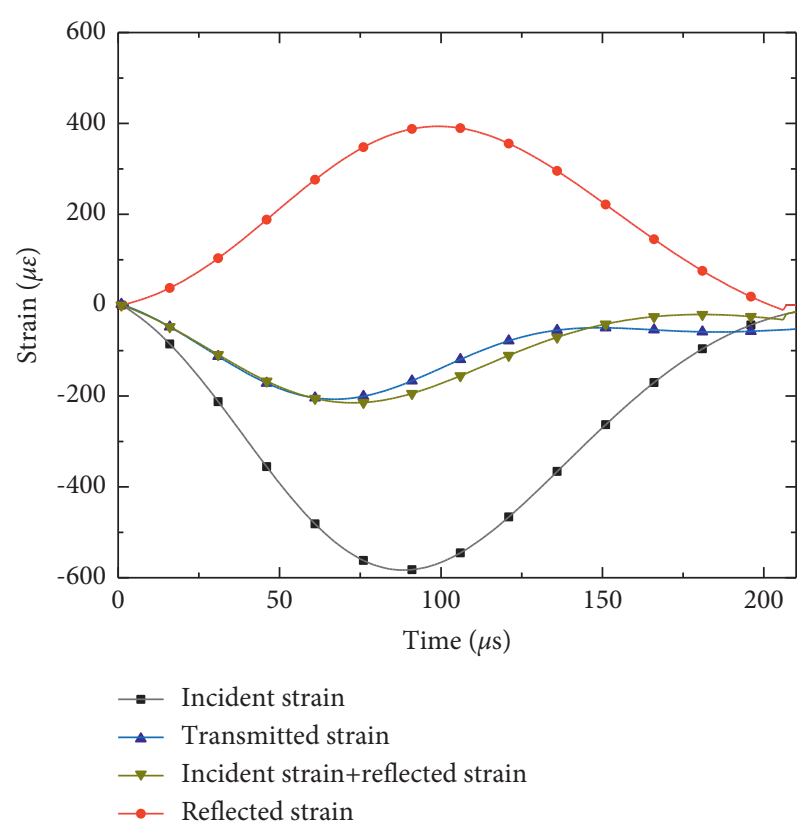

Figure 3: Dynamic stress equilibrium in specimen S-1.

Figure 3 shows a typical stress impulse for specimen S-1. In Figure 3, the $(\mathrm{In}+\mathrm{Re})$ curve is the sum of the incident stress and reflected stress and is approximately equal to the transmitted stress. This result demonstrates that the uniformity of the dynamic stress balance can be achieved during the entire dynamic loading. In this way, the dynamic stress balance was verified.

\section{Main Results}

4.1. Dynamic Stress-Strain Curves. In the experimental dynamics, three specimens were tested for each heating temperature. In each test, using the three-wave analysis method, the average dynamic stress-strain curve of the thermally treated specimen was obtained. Figure 4 shows typical dynamic stress-strain curves of the thermally treated specimen. Observations can be generalized as follows.

(1) Compared with the conventional dynamic stressstrain curve, the dynamic stress-strain curves of the thermally treated sandstone specimens can be approximately divided into three stages: (1) elastic deformation stage, where the stress initially increased linearly in relation to the strain; (2) plastic stage, during which local stress concentration leads to inelastic deformation and crack development and weakens the ability of the specimen to resist failure; and (3) failure and postpeak where specimen failure or instability occurs accompanied by stress drop.

(2) In previous studies, rate-dependent stress-strain curves were categorized into two groups: Class I (where the slope is positive and has an elastic energy release owing to the recovery of the elastic strain) and Class II (the slope of the postpeak stress-strain curve is negative, which means that the elastic energy

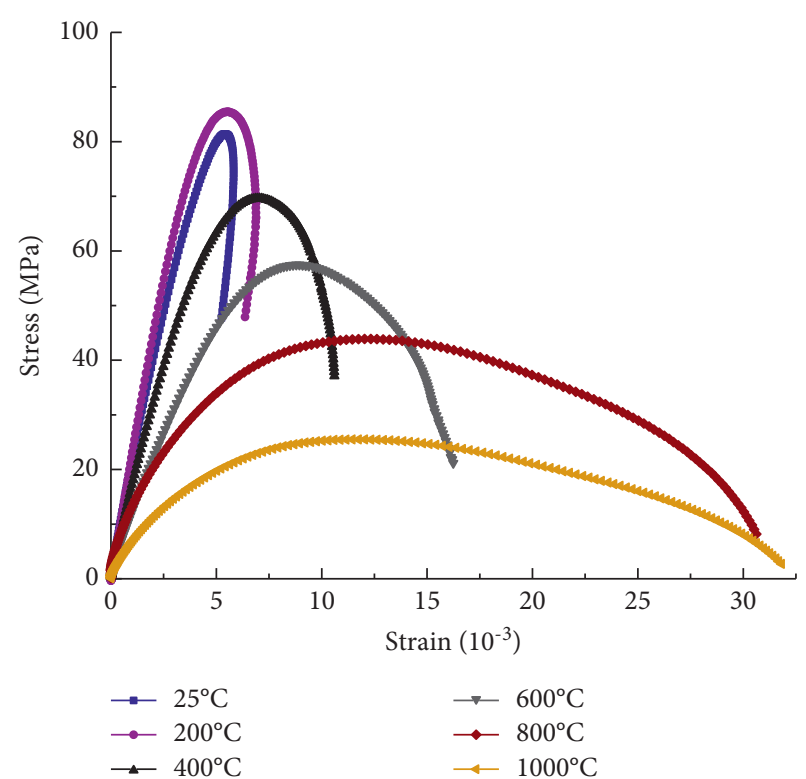

FIgURE 4: Typical dynamic stress-strain curves of the thermally treated specimen.

accumulated in the rock material is sufficient to maintain the entire fracture process). It is also evident that the heating temperatures have significant effects on the dynamic stress-strain curves of the sandstone specimens. Postpeak behavior switched from Class I at a heating temperature of $200^{\circ} \mathrm{C}$ to Class II at heating temperatures above $600^{\circ} \mathrm{C}-1000^{\circ} \mathrm{C}$. In Class I behavior, the slope of the postpeak stress-strain curve increased as the heating temperature $T$ changed from $200^{\circ} \mathrm{C}$ to $400^{\circ} \mathrm{C}$. This change is relevant to the loss of cohesion owing to heating. In the following section, we discuss this change.

4.2. Dynamic Elastic Modulus and Uniaxial Compressive Strength with respect to Heating Temperature. For further analysis, the dynamic elastic modulus and uniaxial compressive strength for each specimen were calculated. The method suggested by Li et al. [20] was used to determine the dynamic elastic modulus, which is defined as the slope of the stress-strain curve in the elastic stage. The correlation between the corresponding parameters of different heating temperatures is plotted in Figure 5. The vertical error bars represent the standard error and indicate the range of the parameters over which the data were averaged. From Figure 5 , the discreteness of the dynamic elastic modulus was not obvious.

At room temperature, the average dynamic elastic modulus was approximately $17.3 \mathrm{GPa}$. It can be seen from Figure 5 that for the same strain rate, the dynamic elastic modulus first increased and then gradually decreased after different temperature treatments. At lower temperatures (up to $200^{\circ} \mathrm{C}$ ), the average dynamic elastic modulus was 21.4 $\mathrm{GPa}$, which is approximately 1.23 times higher than that at room temperature. However, after treatment beyond 


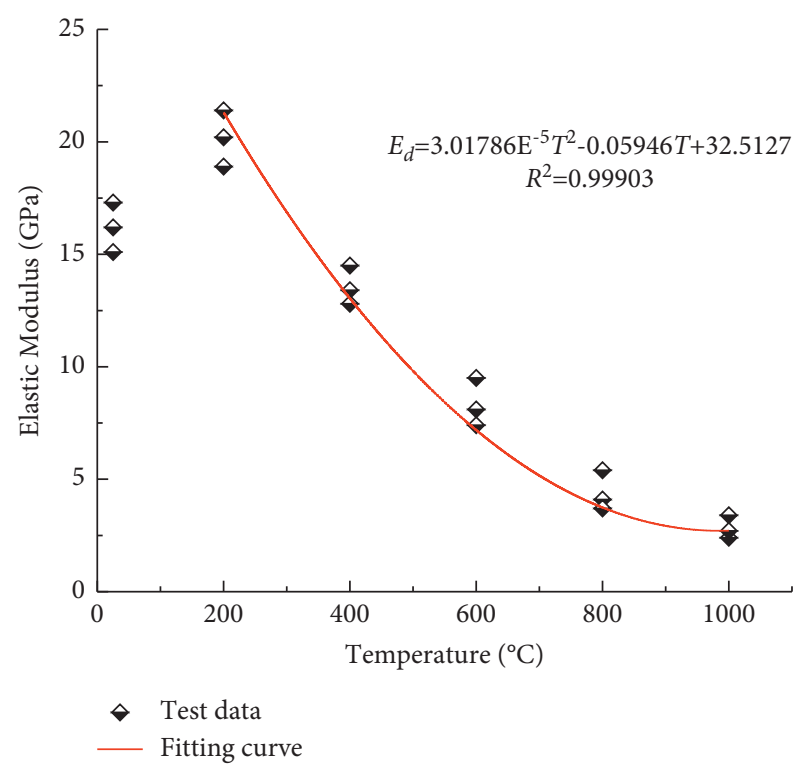

Figure 5: The dynamic elastic modulus of the thermally treated specimen.

$200^{\circ} \mathrm{C}$, the dynamic elastic modulus gradually decreased with the high-temperature treatments. After the $1000^{\circ} \mathrm{C}$ treatments, the dynamic elastic modulus was approximately $3.4 \mathrm{GPa}$, which is approximately $20 \%$ of that at room temperature. A least-square fitted nonlinear function (equation (2) as a function of the heating temperature was used to estimate the expected dynamic elastic response $E_{\mathrm{d}}$ of thermally treated sandstone at $200^{\circ} \mathrm{C}-1000^{\circ} \mathrm{C}$. The correlation coefficient $R^{2}$ was approximately 0.99903 .

$$
E_{\mathrm{d}}=3.01786 E^{-5} T^{2}-0.05946 T+32.5127 .
$$

As shown in Figure 6, between room temperature and $200^{\circ} \mathrm{C}$, the dynamic uniaxial compressive strength showed an approximately proportional change in the temperature treatment. However, between $200^{\circ} \mathrm{C}$ and $1000^{\circ} \mathrm{C}$, the dynamic uniaxial compressive strength gradually decreased. The dynamic uniaxial compressive strength (approximately $37.8 \mathrm{MPa}$ ) was the lowest after treatment at $1000^{\circ} \mathrm{C}$, which was approximately one-fifth of that at room temperature. This phenomenon indicated internal structural changes in sandstone during temperature treatment. A nonlinear fitting was applied to describe the relationship, with a coefficient of determination greater than 0.98267 .

$$
\sigma_{T}=-4.56716 E^{-5} T^{2}-0.01258 T+95.30979 .
$$

4.3. Failure Mode. Since the fracture process of thermally treated sandstone under dynamic loading is very short, a high-frame-rate camera was employed to simultaneously acquire images of fracture processes and further explore their failure mode. Figure 7 shows the failure mode of thermally treated sandstone under SHPB loading.

Figure 7 shows that with the increase in temperature treatments from room temperature and $1000^{\circ} \mathrm{C}$, the failure

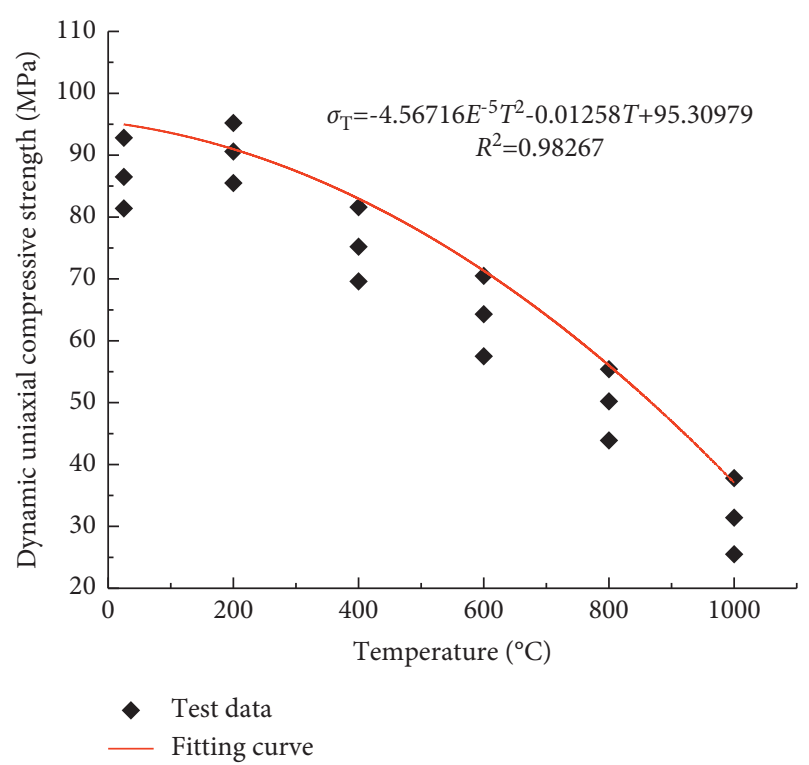

FIGURE 6: The dynamic elastic modulus of the thermally treated specimen.

degree increased. Between room temperature and $400^{\circ} \mathrm{C}$, the thermally treated sandstone specimens behaved as Class I failure, as shown in Figures 7(a) and 7(b). Class I failure is characterized by the recovery of strain in association with stored elastic energy release. After the $200^{\circ} \mathrm{C}$ and $400^{\circ} \mathrm{C}$ treatments, these samples were not in a failure state or spalling-like failure near the free surfaces of the sample, which is the result of the tensile stress reflected from the free boundary. The tests on the other types of rock that were applied to SHPB testing at low strain rates also resulted in similar testing results. However, at treatment beyond $600^{\circ} \mathrm{C}$, samples subjected to dynamic loading were split or fractured, as shown in Figures 7(d)-7(f), which behaves as Class II failure. In this case, the fragment shape was single or double fractured or multiple fragments were induced by lateral splitting. For example, after the $1000^{\circ} \mathrm{C}$ treatments, dynamic loading caused unrecoverable damage to the sample, and this damage was clustered in the microcracks along the loading direction, which was the result of tensile axial splitting. Another typical fracturing feature is that branching cracks are generated near microcracks and then are clustered in the macrofracture network.

Based on previously described results, two failure modes can be interpreted as follows. When the strain rate was low, Class I failure only existed when the heating temperature was no less than $400^{\circ} \mathrm{C}$. Class I failure mostly results from tensile spalling and is the result of the lateral inertia effect since brittle rock material is sensitive to tensile stress, and even very low tensile stress can cause damage. However, the Class II failure mechanism is distinct from lateral inertia effectinduced fracturing usually observed in Class I failure. From the tests, Class II failure mostly results from compressioninduced fracturing with all loss of cohesion. At higher temperatures, more heat-induced microcracks developed in the specimen and showed ductile behavior. With increased microcrack density, it is easier for specimens to dissipate 


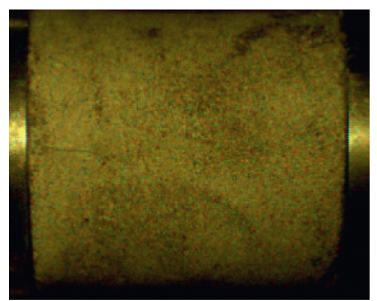

80 us

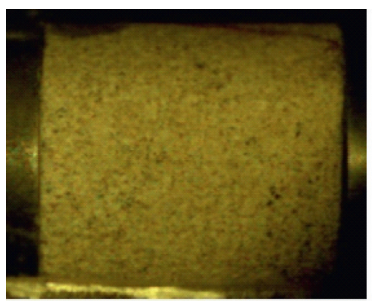

80 us

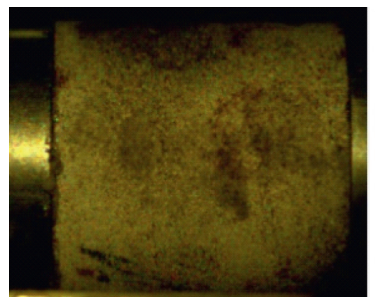

80 us

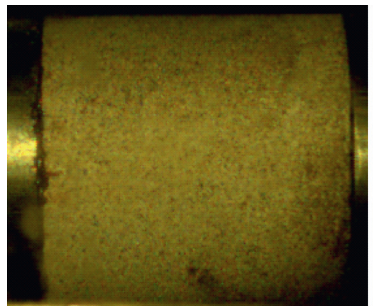

80 us

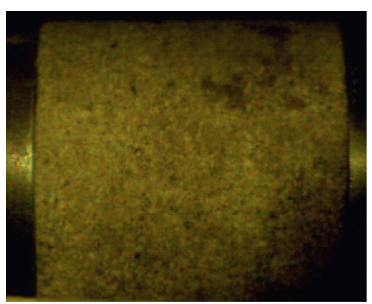

80 us

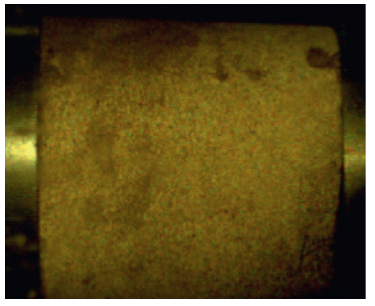

80 us

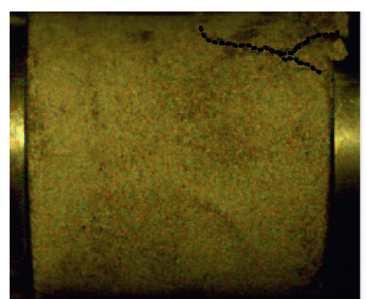

100 us

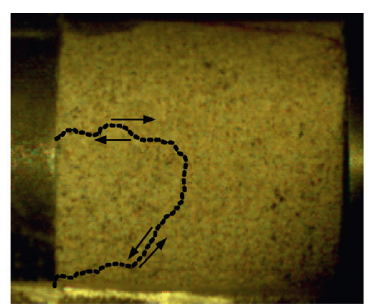

100 us

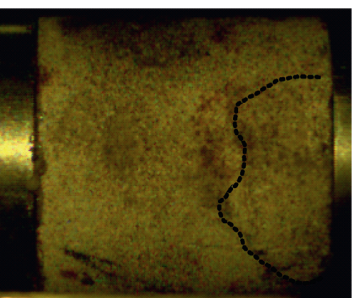

100 us

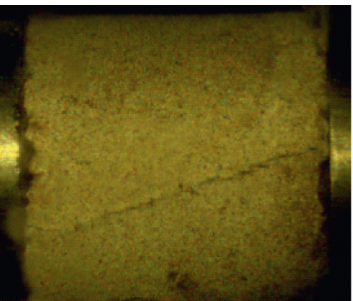

100 us

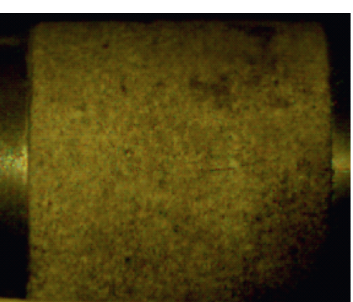

100 us

(e)

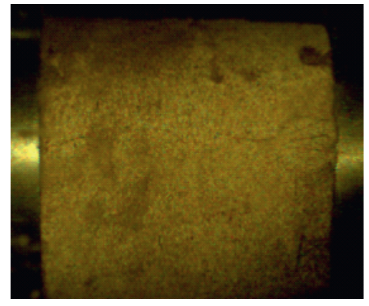

100 us
120 us

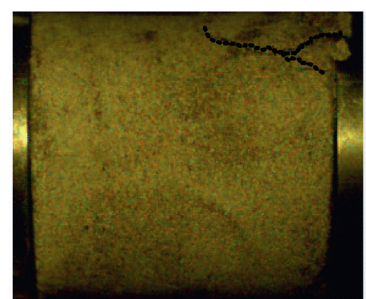

120 us

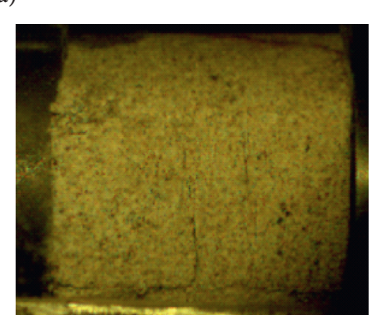

120 us

(b)

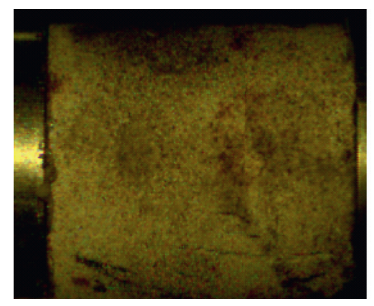

120 us

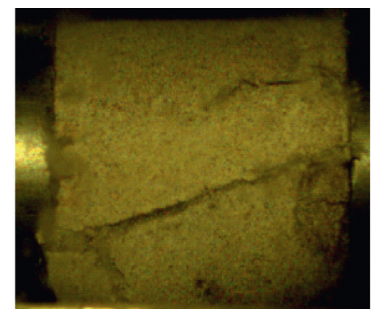

120 us

d)
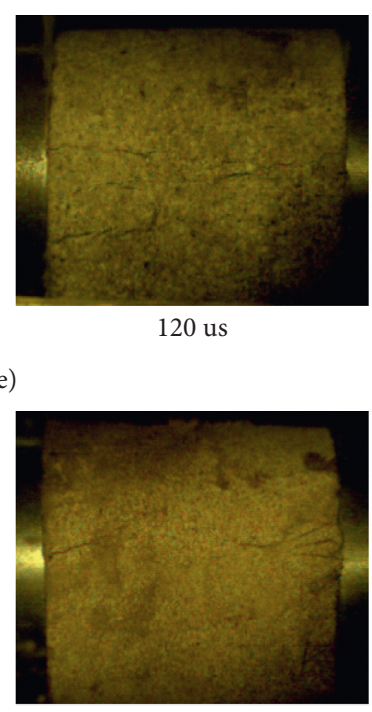

120 us

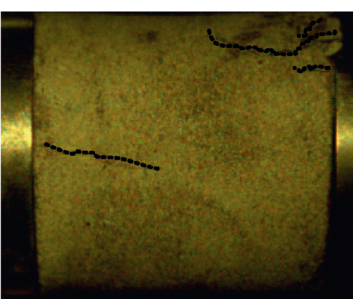

140 us

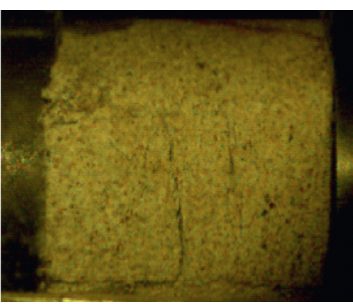

140 us

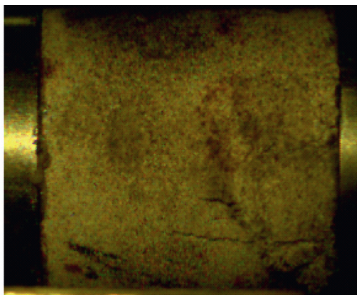

140 us

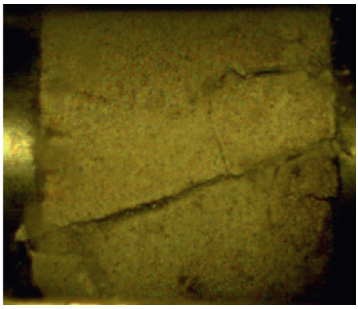

140 us

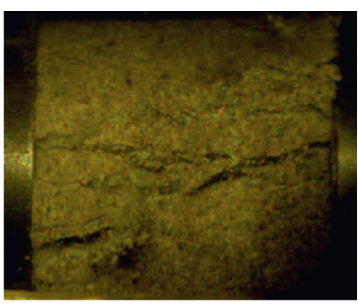

140 us

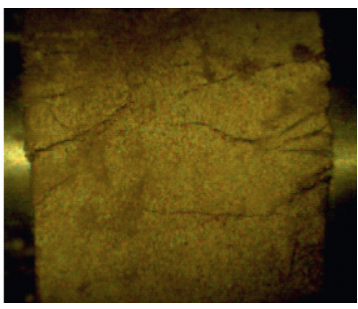

140 us

(f)

Figure 7: Failure mode of thermally treated sandstone under SHPB loading: (a) $25^{\circ} \mathrm{C}$; (b) $200^{\circ} \mathrm{C}$; (c) $400^{\circ} \mathrm{C}$; (d) $600^{\circ} \mathrm{C}$; (e) $800^{\circ} \mathrm{C}$; (f) $1000^{\circ} \mathrm{C}$. 
dynamic loading energy in association with crack propagation, nucleation, and development, which in turn results in macrobehavioral changes.

\section{Discussion}

5.1. Effect of High-Temperature Treatment on Rock Dynamic Mechanical Properties. Rock consists of not only rockforming minerals but also water between or within crystals, including absorbed, bond, and mineral water. Previous studies have shown that the mechanical strength of rocks depends strongly on the density of the cracks and pores. It is also possible that the crack and pore structure of rock are closely related to thermal treatment. Accordingly, the rock dynamic mechanical properties must also change.

In general, the effect of high-temperature treatment on rock was mainly reflected in mineral thermal expansion and thermal reactions. When the temperature increases to $400^{\circ} \mathrm{C}$, the absorbed water is lost, and the bound water and crystal water progressively escape. The remaining pores provide more free space for the thermal expansion of minerals, which closes pores and compacts the rock structure. Due to water loss, the friction between grains increases, which is a contributor to rock strength. After that, the dynamic elastic modulus and uniaxial compressive strength increased.

From the microscopic point of view, the strength of a material depends not only on the stress applied but also on the damage [29]. Some studies have shown that thermally induced damage always incurs structural destruction of minerals due to enhanced thermal reactions (transformation or decomposition). It is noteworthy that thermal reactions of minerals occur at very high temperatures. For instance, Kaolinite was detected up to $450^{\circ} \mathrm{C}$. Quartz experiences $\alpha$-quartz to $\beta$-quartz at $573^{\circ} \mathrm{C}$, which is associated with a volume increase and thermal cracking. Microcracks at the grain boundaries of quartz and feldspar particles were documented at higher temperatures above $750^{\circ} \mathrm{C}$. Progressive transformation $\left(\mathrm{OH}+\mathrm{OH} \rightleftharpoons \mathrm{H}_{2} \mathrm{O}+\mathrm{O}\right)$ of kaolinite takes place at $400-500^{\circ} \mathrm{C}$, which simultaneously results in the formation of amorphous metakaolinite. A mineral component analysis indicated that the sandstone sample was mainly composed of quartz, feldspar, and debris. In the range of $400^{\circ} \mathrm{C}$ up to $1000^{\circ} \mathrm{C}$, this means that most transformations or decomposition of minerals result in thermally induced microcracks. The activation of thermally induced microcracks has negative effects on rock strength.

\subsection{Effect of High-Temperature Treatment on Rock Dynamic} Failure. In conventional loading tests (such as UCS), the failure mode of rock changes from brittle to ductile [30]. At room temperature, a possible brittle rock failure was usually observed, and significant fracture occurred after the peak stress. After the $400^{\circ} \mathrm{C}$ treatment, significant deformation occurred before the final destruction, and failure became obvious. In the SHPB tests, the dynamic failure of thermally treated rock is easily shown to be different from the failure mode of rock subject to quasi-static conditions.
In Section 4.3, it was shown that when the heating temperature was no less than $400^{\circ} \mathrm{C}$, the sandstone specimens exhibited a typical lateral splitting mode (Class I failure). The fracture surface was also found to have very smooth and sharp edges, which were the result of axial splitting failure. As the heating temperatures $T$ changed from $400{ }^{\circ} \mathrm{C}$ to $1000{ }^{\circ} \mathrm{C}$, the sandstone specimens were impacted to small pieces and nearly pulverized (Class II failure). In general, these two classical failure types (Class I and Class II) were observed in the SHPB test. The strain rate was relatively stable in this study so that its effect can be ignored. Based on previously described results, this dynamic fracturing transitioned from single-fractured $\longrightarrow$ fragmented $\longrightarrow$ pulverized when the temperature exceeds the upper threshold. Unfortunately, an accurate upper threshold was not obtained from tests for two reasons. One reason is that a broader temperature interval was set in the tests. Another reason is that the failure mode of rock in the SHPB test was rate dependent. In other words, when the strain rate was different, the upper threshold could change. It is noteworthy that the upper threshold may be $400{ }^{\circ} \mathrm{C}$ when the strain rate was $45.3 / \mathrm{s}$. Class I samples are subject to elastic deformation, which is characterized by unloading after the peak failure is associated with huge elastic strain energy release. These samples are loaded up to a peak well below the quasi-static peak strength, and the sample is not in a failure or roughly fractured state. In contrast to Class I, Class II is subjected to higher heating temperatures with all loss of cohesion and large corrected residual strains. At lower temperatures, the energy consumption required for the heat-induced microcracks to initiate and propagate is limited. The input work by the striker impact is enough to propagate the fracture of the specimen, and another part of the additional elastic energy is released, which behaves as the recovery of the elastic strain. However, at higher temperatures, rock materials contain high thermally induced microcracks that are spaced close enough to each other to interact. At a given strain rate, the input work by the striker impact is capable of activating the thermally induced crack, additional energy will be required to activate secondary cracks, and these secondary cracks nucleate into the fracture surface. This process consumes higher energy and in turn results in a macrofracturing transition.

A comprehensive characterization of the microcracking process can enhance the understanding of rock temperaturedependent failure from a microscopic view. The SEM examination of micrographs showed that the microfracturing mode can be categorized into two main forms: intercrystalline cracks (IE) and intracrystalline cracks (IA), as shown in Figure 8. When the heating temperature was no less than $400^{\circ} \mathrm{C}$, thermal cracking did not occur, and then the sample was not severely damaged. Because the fracturing toughness of transgranular contacts is generally larger than that of intergranular contacts, IE was the chief cracking type in the fracture surface of a specimen fractured by SHPB loading, even though there were some IAs simultaneously. As the heating temperature was changed from $400{ }^{\circ} \mathrm{C}$ to $1000{ }^{\circ} \mathrm{C}$, thermal damage occurred in the samples. Under Class II loading test conditions, IA was the chief cracking form, and 


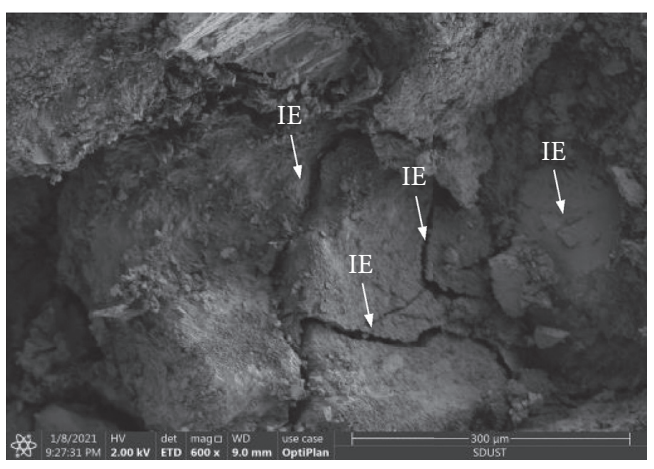

(a)

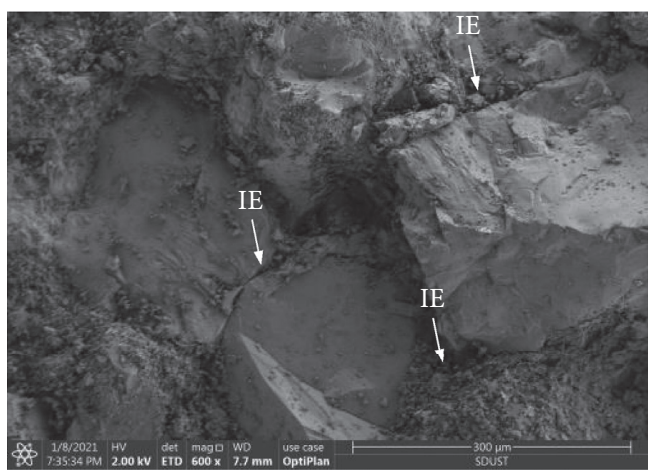

(c)

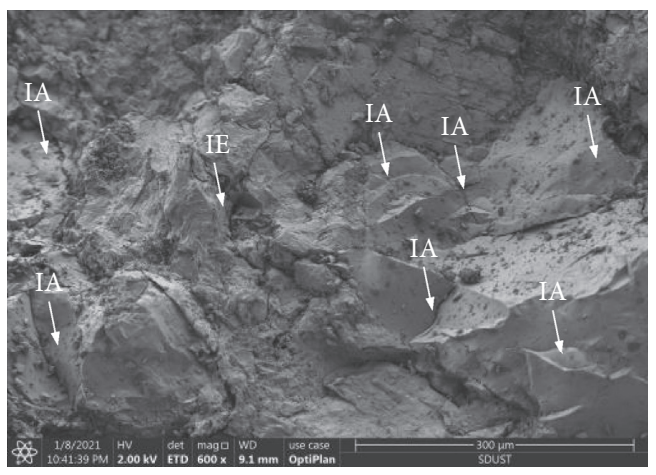

(e)

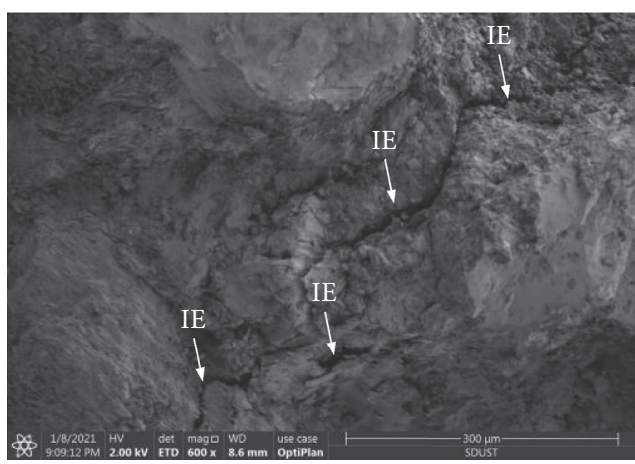

(b)

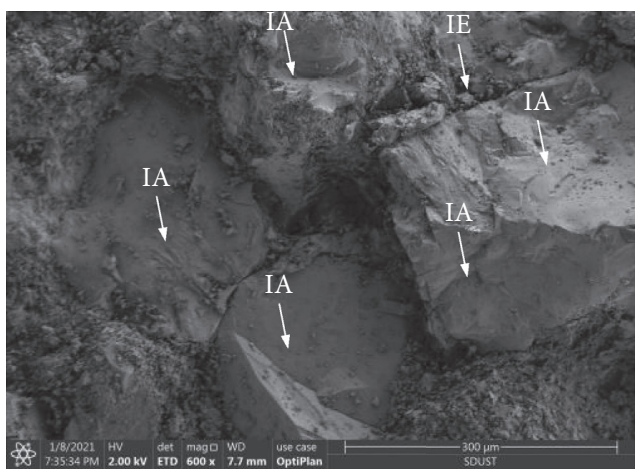

(d)

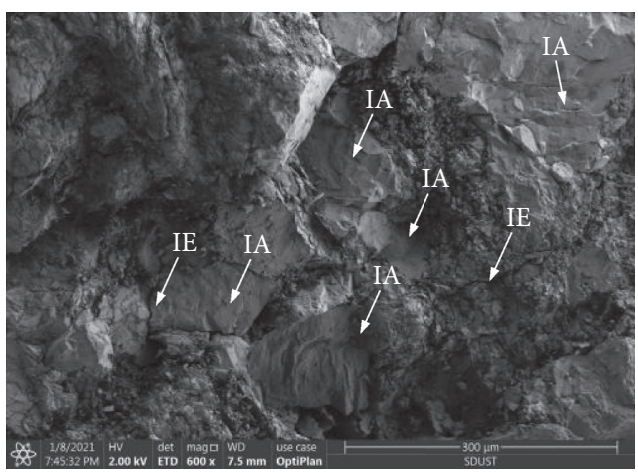

(f)

Figure 8: SEM images of typical medium sandstone fractures at various strain rates: (a) $25^{\circ} \mathrm{C}$; (b) $200^{\circ} \mathrm{C}$; (c) $400^{\circ} \mathrm{C}$; (d) $600^{\circ} \mathrm{C}$; (e) $800^{\circ} \mathrm{C}$; (f) $1000^{\circ} \mathrm{C}$.

IE appeared to be fewer than the former. Because the fracturing toughness of intergranular contacts decreased, the propagating microcracks directly deflected into the interface or penetrated the grain. These cracks commonly nucleate into crack clustering and directly lead to macropulverization phenomena. This type of microcracking process aims to transform the strain energy stored in specimens to dissipated energy (e.g., slipping energy and kinetic energy).

\section{Conclusion}

In this study, typical thermally treated sandstone samples were used to investigate the effect of temperature on the dynamic mechanical behaviors and fracture characteristics of specimens under SHPB tests in the laboratory. The following conclusions can be obtained:
(1) In the SHPB tests, when the heating treatment increased from $200^{\circ} \mathrm{C}$ to $1000^{\circ} \mathrm{C}$, the dynamic uniaxial compressive strength gradually decreased. When the heating temperature did not exceed $200^{\circ} \mathrm{C}$, the dynamic elastic modulus increased; however, in the range of $200^{\circ} \mathrm{C}$ up to $1000^{\circ} \mathrm{C}$, it gradually decreased after different temperature treatments. Finally, two nonlinear fitting formulas were applied to describe the relationship between the dynamic uniaxial compressive strength, the dynamic elastic modulus, and heating treatment temperature.

(2) Based on the results obtained by SHPB tests, the failure mode of heating treatment medium sandstone was characterized by macroscopic end states: intact or single-fractured, fragmented, or pulverized. Then, two classical mechanical types can be 
interpreted as follows: Class I only exists when the heating temperature is less than $200^{\circ} \mathrm{C}$, in which some samples are intact or slightly fractured fragments. In contrast to Class I, Class II samples are subject to a higher heating temperature $\left(400^{\circ} \mathrm{C} \leq T \leq 1000^{\circ} \mathrm{C}\right)$, in which some samples have been sufficiently damaged and become pulverized.

(3) The transition of dominant microcracks from intergranular to transgranular fractures results in different macromechanical types. On the basis of the SEM analysis of thermally treated medium sandstone fractures observed from SHPB tests, intercrystalline cracks were the chief cracking type in Class I tests. In contrast, Class II behavior results from the chief cracking type of intracrystalline cracks.

\section{Data Availability}

The data used to support the findings of this study are included within the article.

\section{Conflicts of Interest}

The author(s) declare that there are no conflicts of interest regarding the publication of this paper.

\section{Acknowledgments}

This research was funded by National Natural Science Foundation of China (nos. 51904163 and 52074170) and Natural Science Foundation of Shandong Province, China (no. ZR2019QEE002).

\section{References}

[1] M. Cai and E. T. Brown, "Challenges in the mining and utilization of deep mineral resources," Engineering, vol. 3, no. 4, pp. 432-433, 2017.

[2] H. Jing, J. Wu, Q. Yin, and K. Wang, "Deformation and failure characteristics of anchorage structure of surrounding rock in deep roadway," International Journal of Mining Science and Technology, vol. 30, no. 5, pp. 593-604, 2020.

[3] S. Fomin, T. Hashida, V. Chugunov, and A. V. Kuznetsov, “A borehole temperature during drilling in a fractured rock formation," International Journal of Heat and Mass Transfer, vol. 48, no. 2, pp. 385-394, 2005.

[4] J. Zhao, "Geothermal testing and measurements of rock and rock fractures," Geothermics, vol. 23, no. 3, pp. 215-231, 1994.

[5] S. Chaki, M. Takarli, and W. P. Agbodjan, "Influence of thermal damage on physical properties of a granite rock: porosity, permeability and ultrasonic wave evolutions," Construction and Building Materials, vol. 22, no. 7, pp. 1456-1461, 2008.

[6] A. Ozguven and Y. Ozcelik, "Effects of high temperature on physico-mechanical properties of Turkish natural building stones," Engineering Geology, vol. 183, pp. 127-136, 2014.

[7] T. B. Yin, Y. Wu, Q. Li, and C. Wang, "Determination of double-K fracture toughness parameters of thermally treated granite using notched semi-circular bending specimen," Engineering Fracture Mechanics, vol. 226, Article ID 106865, 2019.
[8] L. Zhang, X. Mao, R. Liu, X. Guo, and D. Ma, “The mechanical properties of mudstone at high temperatures: an experimental study," Rock Mechanics and Rock Engineering, vol. 47, no. 4, pp. 1479-1484, 2014.

[9] S. Shao, P. G. Ranjith, P. L. P. Wasantha, and B. K. Chen, "Experimental and numerical studies on the mechanical behaviour of australian strathbogie granite at high temperatures: an application to geothermal energy," Geothermics, vol. 54, pp. 96-108, 2015.

[10] Y. Zhao, Z. Wan, Z. Feng, D. Yang, Y. Zhang, and F. Qu, "Triaxial compression system for rock testing under high temperature and high pressure," International Journal of Rock Mechanics and Mining Sciences, vol. 52, pp. 132-138, 2012.

[11] T. F. Wong and W. F. Brace, "Thermal expansion of rocks: some measurements at high pressure," Tectonophysics, vol. 57, no. 2-4, pp. 95-117, 1979.

[12] J. Peng, Z. C. Tang, and D. Hou, "A GSI-softening model for characterizing strength behavior of thermally-damaged rock," Engineering Geology, vol. 292, Article ID 106251, 2021.

[13] J. Liu, W. Shi, and X. Wu, "Experimental study on thermally enhanced permeability of rock with chemical agents," Journal of Petroleum Science and Engineering, vol. 195, Article ID 107895, 2020.

[14] P. Guo, S. C. Wu, G. Zhang, and C. Q. Chu, "Effects of thermally-induced cracks on acoustic emission characteristics of granite under tensile conditions," International Journal of Rock Mechanics and Mining Sciences, vol. 144, pp. 1-3, Article ID 104820, 2021.

[15] J. J. Hu, H. P. Xie, Q. Sun, C. B. Li, and G. K. Liu, "Changes in the thermodynamic properties of alkaline granite after cyclic quenching following high temperature action," International Journal of Mining Science and Technology, vol. 31, no. 6, 2021.

[16] Q. L. Ding, F. Ju, X. B. Mao, D. Ma, B. Y. Yu, and S. B. Song, "Experimental investigation of the mechanical behavior in unloading conditions of sandstone after high-temperature treatment," Rock Mechanics and Rock Engineering, vol. 49, no. 7, pp. 2641-2653, 2016.

[17] S. Q. Yang, P. G. Ranjith, H. W. Jing, W. L. Tian, and Y. Ju, "An experimental investigation on thermal damage and failure mechanical behavior of granite after exposure to different high temperature treatments," Geothermics, vol. 65, pp. 180-197, 2017.

[18] A. M. Ferrero and P. Marini, "Experimental studies on the mechanical behaviour of two thermal cracked marbles," Rock Mechanics and Rock Engineering, vol. 34, no. 1, pp. 57-66, 2001.

[19] L. X. Xiong, H. J. Chen, T. Y. Li, and Y. N. Zhang, "Experimental study on the uniaxial compressive strength of artificial jointed rock mass specimen after high temperatures," Geomechanics and Geophysics for Geo-Energy and Geo-Resources, vol. 4, no. 11, pp. 201-213, 2018.

[20] X. Li, Z. Zhou, T.-S. Lok, L. Hong, and T. Yin, "Innovative testing technique of rock subjected to coupled static and dynamic loads," International Journal of Rock Mechanics and Mining Sciences, vol. 45, no. 5, pp. 739-748, 2008.

[21] D. Li, Z. Han, X. Sun, T. Zhou, and X. Li, "Dynamic mechanical properties and fracturing behavior of marble specimens containing single and double flaws in SHPB tests," Rock Mechanics and Rock Engineering, vol. 52, no. 6, pp. 1623-1643, 2019.

[22] W. Yao, K. W. Xia, and A. K. Jha, "Experimental study of dynamic bending failure of Laurentian granite: loading rate and pre-load effects," Canadian Geotechnical Journal, vol. 56, pp. 228-235, 2018. 
[23] Q. B. Zhang and J. Zhao, "A review of dynamic experimental techniques and mechanical behaviour of rock materials," Rock Mechanics and Rock Engineering, vol. 47, no. 4, pp. 1411-1478, 2014.

[24] X. F. Li, Q. B. Zhang, H. B. Li, and J. Zhao, "Grain-based discrete element method (GB-DEM) modelling of multi-scale fracturing in rocks under dynamic loading," Rock Mechanics and Rock Engineering, vol. 51, no. 12, pp. 3785-3817, 2018.

[25] L. N. Y. Wong, Z. Li, H. M. Kang, and C. I. Teh, "Dynamic loading of Carrara marble in a heated state," Rock Mechanics and Rock Engineering, vol. 50, no. 6, pp. 1487-1505, 2017.

[26] S. Liu and J. Xu, "Study on dynamic characteristics of marble under impact loading and high temperature," International Journal of Rock Mechanics and Mining Sciences, vol. 62, pp. 51-58, 2013.

[27] T. F. Chen, J. Y. Xu, S. Liu, P. Wang, and X. Y. Fang, "Experimental study on dynamic mechanical properties of posthigh-temperature sandstone," Explosion and Shock Waves, vol. 34, no. 2, pp. 195-201, 2014.

[28] Y. X. Zhou, K. Xia, X. B. Li et al., "Suggested methods for determining the dynamic strength parameters and mode-I fracture toughness of rock materials," International Journal of Rock Mechanics and Mining Sciences, vol. 49, pp. 105-112, 2012.

[29] X. Shi, H. Jing, W. Chen, Y. Gao, and Z. Zhao, "Investigation on the creep failure mechanism of sandy mudstone based on micromesoscopic mechanics," Geofluids, vol. 2021, pp. 1-19, Article ID 5550733, 2021.

[30] H. W. Jing, Q. Yin, S. Q. Yang, and W. Q. Chen, "Micromesoscopic creep damage evolution and failure mechanism of sandy mudstone," International Journal of Geomechanics, vol. 21, no. 3, Article ID 04021010, 2021. 